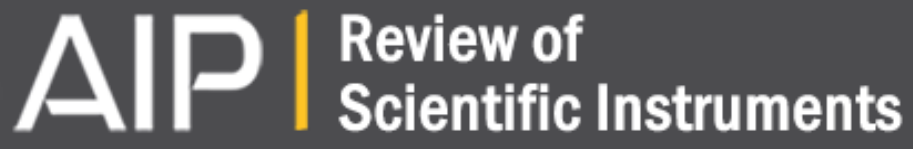

\section{The MAST motional Stark effect diagnostica)}

N. J. Conway, M. F. M. De Bock, C. A. Michael, M. J. Walsh, P. G. Carolan, N. C. Hawkes, E. Rachlew, J. F. G. McCone, S. Shibaev, and G. Wearing

Citation: Review of Scientific Instruments 81, 10D738 (2010); doi: 10.1063/1.3494254

View online: http://dx.doi.org/10.1063/1.3494254

View Table of Contents: http://scitation.aip.org/content/aip/journal/rsi/81/10?ver=pdfcov

Published by the AIP Publishing

\section{Articles you may be interested in}

A non-statistical atomic model for beam emission and motional Stark effect diagnostics in fusion plasmasa)

Rev. Sci. Instrum. 83, 10D504 (2012); 10.1063/1.4728093

Simulation of the motional Stark effect diagnostic gas-filled torus calibrationa)

Rev. Sci. Instrum. 79, 10F523 (2008); 10.1063/1.2969419

Ab initio modeling of the motional Stark effect on MASTa)

Rev. Sci. Instrum. 79, 10F524 (2008); 10.1063/1.2966459

Magnetic fluctuation profile measurement using optics of motional Stark effect diagnostics in JT-60Ua)

Rev. Sci. Instrum. 79, 10F533 (2008); 10.1063/1.2965780

Overview of equilibrium reconstruction on DIII-D using new measurements from an expanded motional Stark effect diagnostica)

Rev. Sci. Instrum. 79, 10F518 (2008); 10.1063/1.2955711

\section{A|P| Journal of}

Journal of Applied Physics is pleased to announce André Anders as its new Editor-in-Chief 


\title{
The MAST motional Stark effect diagnostic ${ }^{a)}$
}

\author{
N. J. Conway, ${ }^{1, b)}$ M. F. M. De Bock, ${ }^{1}$ C. A. Michael, ${ }^{1}$ M. J. Walsh, ${ }^{1, c)}$ P. G. Carolan, ${ }^{1}$ \\ N. C. Hawkes, ${ }^{1}$ E. Rachlew ${ }^{2}$ J. F. G. McCone,${ }^{3}$ S. Shibaev, ${ }^{1}$ and G. Wearing ${ }^{1}$ \\ ${ }^{1}$ EURATOM/CCFE Fusion Association, Culham Science Centre, Abingdon OX14 3DB, United Kingdom \\ ${ }^{2}$ Department of Physics, KTH, EURATOM-VR Association, SE-10691 Stockholm, Sweden \\ ${ }^{3}$ Department of Physics, University College Cork, Association EURATOM-DCU, Cork, Ireland
}

(Presented 18 May 2010; received 16 May 2010; accepted 25 August 2010; published online 28 October 2010)

\begin{abstract}
A motional Stark effect (MSE) diagnostic is now installed and operating routinely on the MAST spherical tokamak, with 35 radial channels, spatial resolution of $\sim 2.5 \mathrm{~cm}$, and time resolution of $\sim 1 \mathrm{~ms}$ at angular noise levels of $\sim 0.5^{\circ}$. Conventional (albeit very narrow) interference filters isolate $\pi$ or $\sigma$ polarized emission. Avalanche photodiode detectors with digital phase-sensitive detection measure the harmonics of a pair of photoelastic modulators operating at 20 and $23 \mathrm{kHz}$, and thus the polarization state. The $\pi$ component is observed to be significantly stronger than $\sigma$, in reasonably good agreement with atomic physics calculations, and as a result, almost all channels are now operated on $\pi$. Trials with a wide filter that admits the entire Stark pattern (relying on the net polarization of the emission) have demonstrated performance almost as good as the conventional channels. MSE-constrained equilibrium reconstructions can readily be produced between pulses.
\end{abstract}

[doi:10.1063/1.3494254]

\section{INTRODUCTION}

The motional Stark effect (MSE) diagnostic ${ }^{1}$ is the principal means of determining the internal magnetic field structure and thus the plasma current distribution in tokamaks. Essentially, the approach relies on the fact that neutral beam atoms moving at high speed through a magnetic field experience a large electric field that induces Stark splitting and thus polarization of the line emission from the atoms. The electric field direction is related to the magnetic field direction (since $\vec{E}=\vec{v} \times \vec{B}$ ), so the measurement of the polarization angle $\gamma$ of the emission can provide spatially localized information about the pitch angle of the magnetic field. This knowledge can be used to constrain an equilibrium reconstruction code such as EFIT (Ref. 2) from which $q$ and current density profiles can be obtained. Commonly, a narrowband interference filter is used to selectively transmit either $\pi$ or $\sigma$ polarized light, which is then analyzed by a polarimeter. Multiple spatial channels $(\geq 10)$ are desirable to usefully constrain EFIT.

The low magnetic field strength in spherical tokamaks (STs), such as MAST and NSTX, makes MSE challenging since the degree of Stark splitting in the emission spectrum is reduced in proportion to the field. (The field in MAST is $\sim 0.5 \mathrm{~T}$ at the magnetic axis and $\sim 0.35 \mathrm{~T}$ at the outboard edge, whereas MSE was developed and proven on devices with fields roughly an order of magnitude higher.) Since the

\footnotetext{
a) Contributed paper, published as part of the Proceedings of the 18th Topical Conference on High-Temperature Plasma Diagnostics, Wildwood, New Jersey, May 2010.

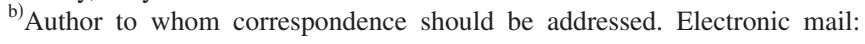
neil.conway@ccfe.ac.uk.

${ }^{c}$ Present address: CHD Department, Diagnostics Division, ITER Organisation, Cadarache, 13106 St. Paul-lez-Durance, France.
}

various factors that broaden the features in the spectrum are to a first approximation unchanged, the Stark pattern can become so blended as to be practically indistinguishable from a single Gaussian (a typical MAST spectrum is shown in Fig. 1). When the $\pi$ and $\sigma$ features of the spectrum overlap strongly, this greatly reduces the attainable polarization fraction, which is important because the angular noise level in the measurement is inversely proportional to the polarization fraction.

In the early years of STs, such as MAST and NSTX, there were doubts that MSE was practical at such low fields. A key issue with adopting the conventional interference filter approach on an ST is that the filter must be much narrower than on high-field machines. For MAST, it was calculated that a full width at half maximum (FWHM) of $\sim 0.1 \mathrm{~nm}$ would be required, and when MAST was built, this was not achievable for a thin-film interference filter of adequate diameter.

A MSE system was then developed for NSTX, which achieved the required FWHM of $0.06 \mathrm{~nm}$ (even smaller than for MAST since it was required to operate at somewhat lower fields) with multistage high-throughput Lyot filters. ${ }^{3}$ By 2004, this system had been partially implemented (with 4 out of the possible 19 spatial channels) and successfully operated, proving the viability of MSE on STs.

When considering how best to implement MSE on MAST, cost was a significant factor. Since the complexity of the Lyot filter makes it expensive, alternatives were sought. When improvements in thin-film interference filter manufacturing made it possible to procure relatively inexpensive narrowband filters with $\sim 0.1 \mathrm{~nm}$ FWHM, $50 \mathrm{~mm}$ diameter and transmission in excess of $50 \%$, it created a potentially costeffective way to proceed. A pilot system ${ }^{4}$ with two spatial channels was designed around the filters and this was oper- 


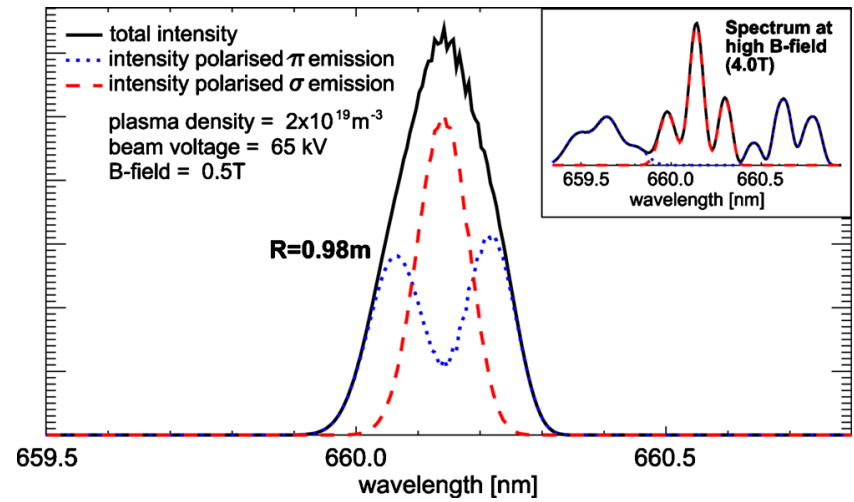

FIG. 1. (Color online) Modeled MSE spectrum typical of MAST (the inset shows how it would look at a field strength of $4 \mathrm{~T}$ ).

ated successfully in 2006. This cleared the way for a fullscale implementation, the design and operation of which are described herein.

In designing the pilot MSE diagnostic, a relatively simple spectral simulation code had been used, but this was known to have limitations, particularly in the treatment of finite collection volume effects. To address this, a new simulation $\operatorname{code}^{5}$ was written to model the spectral broadening effects in a much more complete fashion and calculate the overall system performance. One of the most important results from this code was to highlight the way in which the mixing of nonorthogonal $\sigma$ and $\pi$ emission could change the observed $\gamma$ value by arbitrary amounts. The code provided crucial guidance for the design of the key system elements as described below. De Bock et al. ${ }^{5}$ already summarized the final design parameters of the MAST MSE system-the role of this paper is to elucidate the design choices (some of which underpinned the pilot system itself) and report on the achieved performance of the system along with other operational observations.

\section{SYSTEM DESIGN}

\section{A. Performance targets}

A key performance attribute is the random noise level $\Delta \gamma$ in polarization angle measurements from individual spatial channels at a given time resolution. In designing the system, one question being addressed is at what value of $\Delta \gamma$ further improvements cease to significantly enhance the quality of equilibrium reconstructions. Trials of MSEconstrained EFIT reconstructions, along with consideration of the probable levels of systematic error from various sources, suggested that there would be minimal benefit in achieving $\Delta \gamma$ values of much less than $0.5^{\circ}$. (In perhaps this single respect, MSE is easier on STs due to their high magnetic pitch angles-to achieve equivalent quality on a conventional tokamak would require much smaller $\Delta \gamma$.)

When the performance is shot-noise limited, a quadratic relationship exists between $\Delta \gamma$ and the time resolution $\Delta \mathrm{t}$-i.e., $\Delta \gamma \propto \Delta \mathrm{t}^{-0.5}$. Also, for a given polarization fraction, the time resolution improves linearly with the amount of light collected. As a design guideline, a target time resolution of $\leq 5 \mathrm{~ms}$ was chosen, this being adequately short relative to the energy confinement time and current relaxation time.
The remaining key performance parameter was the spatial resolution. Consideration of the important scale lengths (e.g., of the measured pressure profiles) led to a target of $\sim 20$ spatial channels spanning the region just inboard of the magnetic axis to the outboard edge of the plasma.

These targets were used throughout the design phase, with the simulation code evaluating the performance of each potential configuration.

\section{B. Broadening sources}

The broadening of the Stark pattern (and hence the polarization fraction) is affected by a number of factors. The neutral beam itself plays a major role. The divergence of the beamlets that make up the beam and their geometric "focusing" (whereby the beamlets are individually aimed to converge at a point or on a line) combine to broaden the spectrum very significantly on MAST.

The aperture of the collection optics is the other main source of broadening. This is largely due to the slightly different Doppler shift of the spectrum as observed at different points on the aperture. This effect is dominated by the aperture dimension in the plane containing the line of sight and the beam axis, so the dimension perpendicular to this plane can be extended to gather more light with little additional broadening. This fact was exploited on NSTX, ${ }^{3}$ where a rectangular aperture was used with dimensions of $\sim 20 \times 3 \mathrm{~cm}$. While this approach was considered for MAST, a circular aperture was favored. This is because a drawback of a rectangular aperture is the etendue requirement: comparing an $\mathrm{N} \times 1$ aperture to a circular aperture of unit diameter, the increased solid angle subtended at the collection volume increases the signal by a factor of $\sim(4 \mathrm{~N} / \pi)$, which should improve the noise performance by a factor of slightly over $\mathrm{N}^{0.5}$ ( $\sim 3$ for NSTX) if no additional broadening occurs. However, the etendue requirement increases by a factor of $\mathrm{N}^{2}$ ( 44 for NSTX) for virtually every component in the system. The obvious cost implications, combined with the limited etendue available from interference filters, made this decision quite straightforward.

The dimensions of the collection volume also affect the broadening. However, since good spatial resolution in the radial direction is desirable, the most obvious shape to choose for the cross section of the collection volume is elongated-narrow in the radial direction and similar to the beam width in the other direction-and this shape also minimizes the broadening arising from variations of the Doppler shift. The line of sight orientation (defined by the location of the collection optics) that minimizes broadening is tangential to the flux surfaces where it intersects the beam since this minimizes the variation in magnetic field angles within the collection volume. Again, though, this is also the orientation required for optimal spatial resolution, so no compromises are necessary. (In fact, the chosen collection optics location achieves the tangency condition at a single value of major radius but is sufficiently close to tangency for all radii that there is little variation in spatial resolution.) 


\section{Wavelength matching}

A key design issue was wavelength matching - the provision of some means by which beam voltage changes and filter manufacturing tolerances could be accommodated. For each spatial channel, there is a modest "useful range" around the ideal value for the filter center wavelength (CWL) within which the polarization fraction is acceptably high. On MAST, this useful range is typically of the order of $\pm 0.03 \mathrm{~nm}$ [though slightly larger for $\pi$ components than for $\sigma$ (see Fig. 4 below)].

The accelerating voltage for a neutral beam source can change from time to time (e.g., to modify the injected power, or if a beamline has not been reconditioned to full nominal voltage after maintenance work). Since the Doppler shift of the light from the beam neutrals varies with the voltage, the MSE diagnostic should ideally be able to cope with some reasonable wavelength variation (typically a $\Delta \mathrm{V}$ of only $1 \mathrm{kV}$ corresponds to a $\Delta \lambda$ of $\sim 0.03 \mathrm{~nm}$ ). The stated CWL manufacturing tolerance for the interference filters was $\pm 0.02 \mathrm{~nm}$.

On tokamaks that employ relatively wide filters, an adequate amount of fine tuning can normally be obtained by tilting the filters slightly, which blueshifts the bandpass region. However, when very narrow filters are tilted, even when illumination solid angles are modest, the bandshape quickly collapses. The very limited tuning range achievable makes this scheme unattractive for MAST.

Several schemes to address this issue were considered and rejected. One promising scheme was based on thermal tuning of the filter wavelengths (as on JT-60U), which would in principle allow completely automatic control.

Another more pragmatic scheme was also considered: to simply "repatch" the detector end of the system, i.e., to shift fiber bundles to alternative filterscopes providing the best wavelength match. Clearly, this approach does not provide a continuously variable CWL setting for each channel. Also, the $\Delta \lambda$ corresponding to a change of beam voltage is not equal for all channels (being smallest on the outboard side, where the Doppler shift is least due to the views making the largest angle with the beam axis). Nonetheless, the simplicity of this approach held considerable appeal.

However, with the originally planned number of spatial channels (20), the wavelength steps between channels were relatively coarse - considerably bigger than the useful wavelength range - and this would have meant that many beam voltage settings did not result in good wavelength matches for most spatial channels. One solution to this was to reduce the wavelength step by dividing the collection region into more spatial channels. It was determined that with 35 spatial channels, the interchannel wavelength steps became small enough to permit good matching for any anticipated beam voltage.

Performance modeling (backed up by the results from the pilot system) of the 35-channel system indicated that the angular noise and time resolution targets could still be met, despite the smaller per-channel etendue. The falloff in performance with etendue was less severe than might be expected since a reduced collection volume suffers less from broadening effects and thus has a higher overall polarization fraction. A welcome side-effect of the increased channel count is of course enhanced spatial resolution.

Since the thermal tuning system-despite its appealwould clearly have added significant cost and development time, the decision was made to adopt the 35-channel system tuned by repatching. (However, components were designed to facilitate possible retrofitting of a thermal tuning system in the future.) A set of 42 filterscopes houses the filters for the 35 channels, with the subset in use at any moment being dependent on the beam voltage.

\section{Components}

This section highlights other design considerations for key elements of the system.

\section{Photoelastic modulators}

A module containing a pair of photoelastic modulators (PEMs) plays a key role in measuring the polarization state of the light. Each PEM contains a fused-silica plate in which a mechanical oscillation produces birefringence, with the retardance commonly set at $\pm 180^{\circ}$ (i.e., \pm half a wave). The PEMs are mounted with their axes $45^{\circ}$ apart and are followed by a linear polarizer set at $22.5^{\circ}$ from each PEM. This arrangement encodes polarization information as intensity modulation in the transmitted light, ${ }^{6}$ which can then be coupled into optical fibers (which do not preserve polarization) and brought outside the tokamak radiation shielding to the sensitive optical detectors.

The PEMs operate at different frequencies, 20 and $23 \mathrm{kHz}$ for the module used on MAST (similar to that used on many other tokamaks), and $\gamma$ is usually obtained from the ratio of the amplitudes of the second harmonic of each PEM, i.e., 40 and $46 \mathrm{kHz}$ (the PEM retardances must also be known). In the synchronous detection of these amplitudes, a bandwidth limit arises, related to the frequency gap around each harmonic in the power spectrum (which contains multiple harmonics of each PEM as well as intermodulation products). Effectively, this means that the maximum bandwidth at which $\gamma$ can be determined is $\sim 1.5 \mathrm{kHz}$, irrespective of the amount of light detected. If the PEM retardances are set somewhat higher than usual at $\sim 220^{\circ}$, where the first harmonics of the PEMs are suppressed, this removes the intermodulation product at $43 \mathrm{kHz}$ and permits the demodulation bandwidth for the second harmonics to be increased to $3 \mathrm{kHz}$. This is the normal operating mode on MAST.

The useful clear aperture of the PEMs is nominally $56 \mathrm{~mm}$ (see test results below), and almost all of this is used on MAST, while the acceptance angle range is nominally $> \pm 20^{\circ}$, about half of which is used in the horizontal plane, with much less used in the vertical plane.

\section{Detectors}

Off-the-shelf detector modules were used, incorporating a $3 \mathrm{~mm}$ diameter avalanche photodiode (APD) sensor, a lownoise amplifier, and an on-board high-voltage power supply which means the modules need only a $5 \mathrm{~V}$ DC input voltage (it was found that power supply noise coupled readily to the signal output, so low-noise linear supplies were used). 
One minor modification was requested to the standard modules that had been used in the pilot system: increased bandwidth. The principal requirement is to measure the second harmonics of the PEM frequencies in order to determine $\gamma$. However, by measuring the fourth harmonics too, it is possible to independently monitor the retardances of both PEMs on every pulse. This provides protection against systematic errors or drifts. The detector bandwidth required for the fourth harmonics is thus $\sim 92 \mathrm{kHz}$. In order to accommodate this without significant attenuation or phase shift, the APD amplifier bandwidth ( $3 \mathrm{~dB}$ point) was extended from $100 \mathrm{kHz}$ to approximately $250 \mathrm{kHz}$ (the APD outputs are digitized at $1 \mathrm{MHz}$ by 14-bit ADCs). The amplitudes of the fourth harmonics also improve the measurement of $\gamma$, particularly when the PEM retardances are set to $220^{\circ}$ as mentioned above (as this strengthens the fourth harmonics).

\section{Collection optics and view location}

The collection lens used is identical to that used on the upgraded MAST CXRS (Ref. 7) diagnostic, with $72 \mathrm{~mm}$ focal length and $38 \mathrm{~mm}$ aperture. Its compatibility with MSE was by design, since when the CXRS upgrade was implemented in 2004, the tolerable aperture size for MSE on MAST was already relatively clear, and therefore the lens design was constrained accordingly.

It was originally expected that the MSE implementation would in fact share an off-midplane CXRS view, combining the optical fibers for both diagnostics on the same focal plane. However, when this view location was modeled with the new simulation code, it was found that the detected $\gamma$ value would vary significantly ${ }^{5}$ with wavelength (more than $2^{\circ}$ within the useful wavelength range for most channels). This would have introduced undesirably large systematic uncertainties in the $\gamma$ measurements. An alternative location on the midplane was found to exhibit minimal sensitivity to this effect and was therefore selected.

\section{Fiber bundles}

The fiber bundles (see schematic in Fig. 2) have 19 fibers per spatial channel to permit tight hexagonal packing at the filterscope end of the bundle, minimizing the solid angle with which the filter is illuminated and thus the consequent broadening of the filter bandshape. The fibers are also mapped such that those imaged nearest to the neutral beam axis (where the emission is brightest) are placed nearest to the center of the hexagonal pattern, as this minimizes the impact of any bandshape broadening. Polymer-clad fibers with $400 \mu \mathrm{m}$ core diameter and a numerical aperture of 0.37 were used. The bundles are $\sim 35 \mathrm{~m}$ long and were built as modules of five spatial channels each to simplify the construction (a small amount of dead space between modules was unavoidable). In total, eight modules were actually installed (i.e., 40 spatial channels) to allow spare capacity in case of breakage.

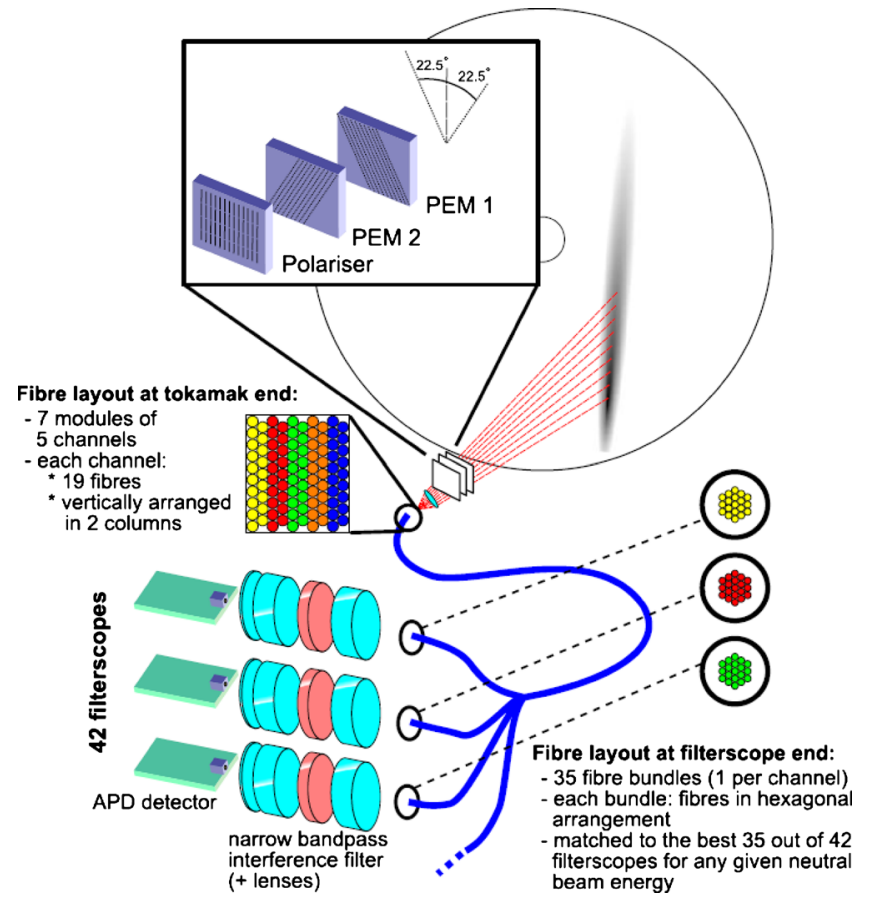

FIG. 2. (Color online) Schematic of the MAST MSE system.

\section{Filterscopes}

The "filterscopes" image the fiber bundles onto the APD detectors with a collimated region in between where the light is passed through the interference filter. Off-the-shelf achromatic doublets and meniscus lenses were used. Although the light is injected into the fibers at $\sim \mathrm{f} / 2$ at the tokamak end, it is extracted at a higher solid angle by the filterscope, $\sim \mathrm{f} / 1.5$. This helps recover some of the light that has "diffused" in angular terms out of the injection solid angle over the length of the fibers. It is estimated that this provides $\sim 20 \%$ more signal. Although the fiber bundle diameter is only $\sim 2.2 \mathrm{~mm}$, its image on the $3 \mathrm{~mm}$ diameter APD is slightly smaller still to help ensure that small position mismatches do not cause a loss of signal (which would be localized to one part of the collection volume and could thus introduce systematic errors).

The central cell that houses the bandpass filter was designed to be removed easily, both to simplify filter testing/ calibrations and to facilitate the possible implementation of a thermal tuning upgrade in the future. The fiber bundle connection to the filterscope is also readily removed when repatching the bundles to alternative filterscopes. The compact design allows all of the 42 filterscopes to be housed in a single 19 in. cubicle ( $42 \mathrm{U}$ in height).

\section{Filters}

The narrowband filters were originally intended to be of two-cavity design but manufacturing proved more difficult and costly than expected. A single cavity design was then subjected to bandshape tests and performance modeling and found to be acceptable. Transmission is typically $\sim 60 \%$.

The target FWHM for the filters was set at $0.12 \mathrm{~nm}$, slightly higher than the absolute minimum believed to be practical $(0.1 \mathrm{~nm})$. This was because modeling showed that 
the signal quality would be almost identical at 0.1 and $0.12 \mathrm{~nm}$, and it was expected that the wider filters could be more easily manufactured (and have slightly better transmission). The filters were also designed to have a high thermal coefficient (more than double the normal value for this type of filter) to facilitate thermal tuning if implemented. The finite solid angle subtended by the fiber bundle at the filter (corresponding to $\sim \mathrm{f} / 35$ ) increases the FWHM by less than $5 \%$.

\section{Others}

The vacuum window for the diagnostic view is mounted on a gate valve, which makes it possible to remove the window for testing or cleaning and which also acts as a shutter to prevent deposition on the window during interpulse glowdischarge cleaning. The walls of the MAST vessel are for the most part sprayed with a layer of colloidal graphite to reduce reflections. The MSE lines of sight intersect various parts of the wall as well as one of the carbon fiber composite beam dumps (for one of the heating beams). Reflected light is therefore not believed to be a problem.

A polymer film polarizer is used, which absorbs the rejected polarization and transmits $\sim 38 \%$ of unpolarized light. Higher efficiency metal polarizers are available (with close to $50 \%$ transmission for unpolarized light) and were considered, but it was felt that systematic errors might arise through secondary reflections of the rejected polarization.

A high-resolution spectrometer fitted with a CCD camera was procured as part of the system and is used to characterize the bandpass filters. It can also measure the full beam-emission spectrum for up to five spatial channels at a time.

\section{OPERATION}

\section{A. Commissioning}

Bench characterization and calibrations of the PEMs revealed significant spatial variations (well in excess of $40^{\circ}$ ) in the retardance for both PEMs within the nominal clear aperture of $56 \mathrm{~mm}$. To some extent, these variations will average out but it is possible that they could produce a systematic effect (e.g., if the collection volume is influenced nonuniformly) and further modeling may be carried out to investigate their impact. Also, the measured retardances did not in general match the selected retardance at the controller. However, the variations of the retardance with angle of incidence were found to be very small and are effectively negligible for the range of angles used on MAST. The stability of the retardance over time appears to be excellent so far.

Spatial calibrations of the system have been performed via backprojection of light through the optical fibers onto the vessel wall with a small aperture inserted at the collection lens pupil (just in front of the lens). Calibrations of the angular response of the system are performed from time to time without the plasma by firing the beam into the vessel with a small amount of neutral deuterium gas present, using varying combinations of toroidal and poloidal fields. At moderate to high gas densities, the measured angles vary with density (believed to be due to reneutralization of fast beam ions ${ }^{8}$ ),

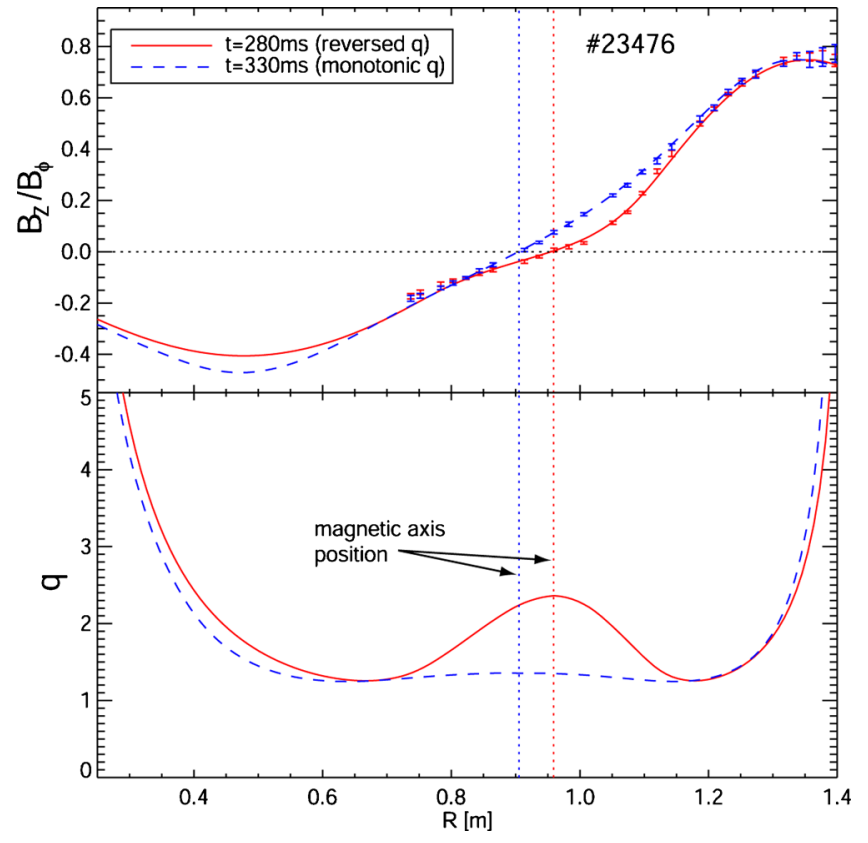

FIG. 3. (Color online) Typical results. The upper panel shows experimental points overlaid with fits from MSE-constrained EFIT. The lower panel shows the corresponding $q$ profiles.

but at low enough densities $\left(<5 \times 10^{18} \mathrm{~m}^{-3}\right)$, this effect is absent and the measurement is believed to be uncontaminated. Bench calibrations have also been performed using a polarizer mounted on a rotating stage.

One channel has recently been fitted with a trial thermal tuning system and this is working well; no decision has yet been made on extending thermal tuning to all channels.

\section{B. Performance}

The system performance has comfortably exceeded the design targets. For typical shots, around 15 out of the 35 channels have adequate signal quality to meet the $\Delta \gamma$ $\leq 0.5^{\circ}$ target at $\Delta \mathrm{t}=0.5 \mathrm{~ms}$, with most of the others meeting it at $\Delta \mathrm{t}=1 \mathrm{~ms}$ (and all channels with $\Delta \mathrm{t} \leq 2 \mathrm{~ms}$ ). The spatial resolution is $\sim 2.5 \mathrm{~cm}$. Some sample results are shown in Fig. 3. In this example, MSE results initially show reversed magnetic shear, which is then lost as a result of an instability shortly before the end of the discharge.

\section{Enhanced $\pi / \sigma$ ratio}

Initially, most channels were configured to measure $\sigma$-polarized emission, as this was expected to provide the lowest $\Delta \gamma$ values. However, it was noted that polarization angles measured during beam-into-gas calibration pulses differed by roughly $90^{\circ}$ from the angles measured during beaminto-plasma pulses. Upon investigation, it was realized that the $\sigma$ emission was at all times significantly weaker than the $\pi$ emission, and that this effect was much more pronounced in the beam-into-gas pulses such that the $\pi$ emission dominated at all wavelengths (which caused the observed angle change). This is caused by nonstatistical populations in the excited states of the beam atoms. In hindsight, this should probably have been anticipated, having been observed ${ }^{9}$ on JET in 1989 (though observations on TFTR were consistent ${ }^{10}$ 


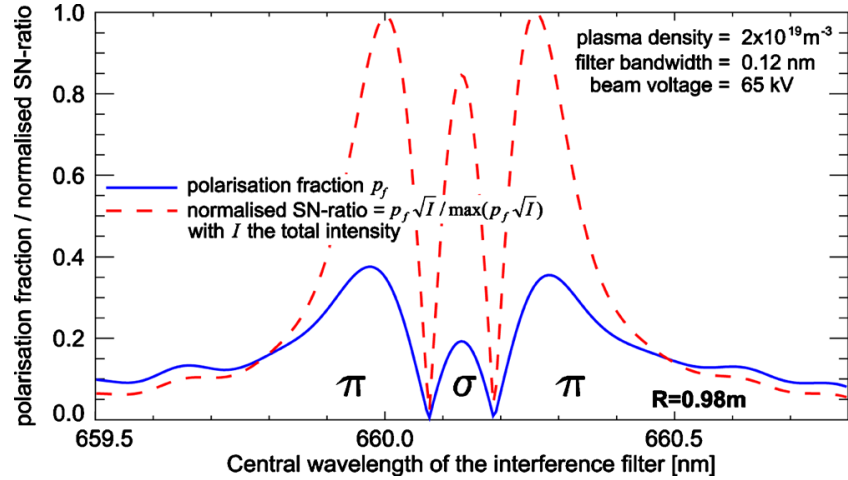

FIG. 4. (Color online) Modeled performance with enhanced $\pi / \sigma$ ratio. The polarization fraction is much higher for $\pi$ than $\sigma$, but the SNR is only slightly higher due to the higher total intensity in the center of the Stark pattern.

with statistical populations). Theoretical treatments of the $\pi / \sigma$ imbalance exist ${ }^{9,11}$ (Ref. 9 treats only the beam-intoplasma case, while the more recent work in Ref. 11 also treats the beam-into-gas case) and these have been compared with the results from MAST. There is reasonable agreement between experiment and theory, but the density dependence predicted by the modeling does not so far appear to match the experimental data. More work on this will be required. Overall, $\sigma$ is effectively weakened by $\sim 20 \%$ in the plasma case and $\sim 50 \%$ in the gas case.

The $\pi / \sigma$ ratio is increased sufficiently in plasma pulses to make the signal to noise ratio (SNR) slightly better on $\pi$, so this is how most channels are now configured. This can be seen in Fig. 4, which also shows why the useful $\lambda$ range is wider for $\pi$ than $\sigma$.

Furthermore, when the net polarization fraction of the entire Stark pattern was calculated, it was apparent that while significantly lower than can be achieved with a narrow filter (the increased light intensity offsets this to some extent), it remains usefully high $(5 \%-10 \%)$. As a result, trials were performed using a much wider filter (1 nm FWHM) with encouraging results-the noise level was almost as good as for the conventional channels and the measured $\gamma$ values matched well with neighboring channels. This approach would probably not be feasible on a high-field machine due to overlap between the Stark patterns from the full and halfenergy beam components, and indeed at sufficiently high plasma density the level populations should become almost fully statistical. Also, a wide filter admits more background emission (e.g., bremsstrahlung) and if any of this emission were to be partly polarized (e.g., by reflections from machine components), it could disturb the measurement. Nonetheless, this may be a useful technique in some situations, and further trials seem worthwhile.

\section{ACKNOWLEDGMENTS}

This work was funded partly by the United Kingdom Engineering and Physical Sciences Research Council under Grant No. P/G003955 and by the European Communities under the contract of association between Euratom and CCFE. The views and opinions expressed herein do not necessarily reflect those of the European Commission.

${ }^{1}$ F. M. Levinton, R. J. Fonck, G. M. Gammel, R. Kaita, H. W. Kugel, E. T. Powell, and D. W. Roberts, Phys. Rev. Lett. 63, 2060 (1989).

${ }^{2}$ L. C. Appel, G. T. A. Huysmans, L. L. Lao et al., Proceedings of the 33rd EPS Conference on Plasma Physics and Controlled Fusion, 2006, Paper No. EFDA-JET-CP(06)03-07, p. 2.184 (http://www.iop.org/Jet/fulltext/ EFDC060307.pdf).

${ }^{3}$ F. M. Levinton and H. Yuh, Rev. Sci. Instrum. 79, 10F522 (2008).

${ }^{4}$ M. Kuldkepp, M. J. Walsh, P. G. Carolan, N. J. Conway, N. C. Hawkes, J. McCone, E. Rachlew, and G. Wearing, Rev. Sci. Instrum. 77, 10E905 (2006).

${ }^{5}$ M. F. M. De Bock, N. J. Conway, M. J. Walsh, P. G. Carolan, and N. C. Hawkes, Rev. Sci. Instrum. 79, 10F524 (2008).

${ }^{6}$ M. Kuldkepp, N. C. Hawkes, E. Rachlew, and B. Schunke, Appl. Opt. 44, 5899 (2005)

${ }^{7}$ N. J. Conway, P. G. Carolan, J. McCone, M. J. Walsh, and M. Wisse, Rev. Sci. Instrum. 77, 10F131 (2006).

${ }^{8}$ H. Y. Yuh, F. M. Levinton, S. D. Scott, and J. Ko, Rev. Sci. Instrum. 79, 10F523 (2008).

${ }^{9}$ A. Boileau, M. von Hellermann, W. Mandl, H. P. Summers, H. Weisen, and A. Zinoviev, J. Phys. B 22, L145 (1989).

${ }^{10}$ F. M. Levinton, Rev. Sci. Instrum. 70, 810 (1999).

${ }^{11}$ M. F. Gu, C. T. Holcomb, R. J. Jayakuma, and S. L. Allen, J. Phys. B 41, $095701(2008)$ 